\title{
Distinguishing highly-related outbreak-associated Clostridium botulinum type $A(B)$ strains
}

\author{
Brian H Raphael*, Timothy B Shirey, Carolina Lúquez and Susan E Maslanka
}

\begin{abstract}
Background: In the United States, most Clostridium botulinum type A strains isolated during laboratory investigations of human botulism demonstrate the presence of an expressed type A botulinum neurotoxin (BoNT/ A) gene and an unexpressed BoNT/B gene. These strains are designated type A(B). The most common pulsed-field gel electrophoresis (PFGE) pattern in the C. botulinum PulseNet database is composed of A(B) strains. The purpose of this study was to evaluate the ability of genome sequencing and multi-loci variable number of tandem repeat analysis (MLVA) to differentiate such strains.

Results: The genome sequences of type A(B) strains evaluated in this study are closely related and cluster together compared to other available C. botulinum Group I genomes. In silico multilocus sequence typing (MLST) analysis (7-loci) was unable to differentiate any of the type $A(B)$ strains isolated from seven different outbreak investigations evaluated in this study. A 15-locus MLVA scheme demonstrated an improved ability to differentiate these strains, however, repeat unit variation among the strains was restricted to only two loci. Reference-free single nucleotide polymorphism (SNP) analysis demonstrated the ability to differentiate strains from all of the outbreaks examined and a non-outbreak associated strain.
\end{abstract}

Conclusions: This study confirms that type A(B) strains that share the same PFGE pattern also share closely-related genome sequences. The lack of a complete type $A(B)$ strain representative genome sequence hinders the ability to assemble genomes by reference mapping and analysis of SNPs at pre-identified sites. However, compared to other methods evaluated in this study, a reference-free SNP analysis demonstrated optimal subtyping utility for type A(B) strains using de novo assembled genome sequences.

Keywords: Botulism, Pulsed-field gel electrophoresis, Multi-loci variable number of tandem repeat analysis, Comparative genomic analysis, Single nucleotide polymorphism

\section{Background}

Botulism is a paralytic disease caused by the action of botulinum neurotoxins (BoNT) at neuromuscular junctions. BoNTs are produced by various botulinum toxin producing clostridia (BTPC) including Clostridium botulinum and some strains of C. butyricum and C. baratii [1]. Botulism can occur when food contaminated with BoNT is ingested or when organisms producing BoNT colonize the intestine (eg. infant botulism, adult intestinal colonization botulism) or when these organisms infect and produce toxin in wounds (eg. wound botulism due to traumatic injury or injection drug use) [1].
C. botulinum are classified into four Groups (I-IV) which are distinguished by biochemical properties and phylogenetic differences [2]. All strains of C. botulinum that produce BoNT/A belong to Group I. Nucleotide sequencing of the genes encoding BoNT/A reveals the presence of several toxin subtypes (eg. A1-A5) which form unique clades [3-5]. Some type A strains that produce BoNT/A also harbor an unexpressed gene encoding BoNT/B and are designated type A(B) [6].

Between 2010 and 2013, 47 C. botulinum type A strains were isolated during 36 laboratory investigations of botulism at the Centers for Disease Control and Prevention

\footnotetext{
* Correspondence: BRaphael@cdc.gov

Enteric Diseases Laboratory Branch, Centers for Disease Control and Prevention, Atlanta, GA 30329, USA
} 
(CDC). Among these strains, $86 \%$ were determined to possess an unexpressed BoNT/B gene suggesting that type $\mathrm{A}(\mathrm{B})$ strains are common among US botulism cases (unpublished data). The bont/A1 nucleotide sequence associated with type $A(B)$ strains is highly conserved and contains only two nucleotide substitutions compared to the bont/A1 sequence found in type A strains without an unexpressed bont/B [3]. A focused microarray featuring selected oligonucleotide probes based on the C. botulinum type A strain ATCC3502 genome sequence was unable to distinguish a group of unrelated type $A(B)$ strains which suggested that these strains may share a high degree of genomic content [7]. Using a larger DNA microarray featuring probes targeting coding sequences in the ATCC3502 genome sequence, Carter et al. [4] demonstrated that several type A(B) strains isolated in North America shared a high degree of genomic content. Analysis of diverse panels of C. botulinum strains using multi-loci variable number of tandem repeat analysis (MLVA) and amplified fragment length polymorphism (AFLP) demonstrate that type A(B) strains cluster separately from strains encoding other bont/A subtypes and from strains expressing BoNT/A1 but lacking an unexpressed bont $/ B[3,8]$. More recently, a standardized C. botulinum pulsed-field gel electrophoresis (PFGE) method has been established (http://www.cdc.gov/ pulsenet/PDF/c-botulinum-protocol-508c.pdf). A national subtyping database (PulseNet) of PFGE patterns using this method is maintained at CDC and individual patterns are given unique identifiers. The most frequent SmaI PFGE pattern (30\%) observed among 256 C. botulinum types A, B, E, and F strains examined has been designated DRPS16.0001. Additional characterization of these strains revealed that they are type $\mathrm{A}(\mathrm{B})$ and all share the same PFGE XhoI pattern (DRPX11.0001).

During laboratory investigations of botulism, specimens from different sources may be examined. For instance, in a foodborne botulism outbreak, clinical samples (such as serum and stool) from one or more individuals as well as suspected food sources may be submitted for testing. Toxin detection either in clinical samples or food ingested by individuals with clinical symptoms of botulism is sufficient for laboratory confirmation [1]. Additionally, BTPC may also be isolated from both stool and food. Isolation of BTPC from stool of individuals with botulism provides ancillary evidence of toxin serotype when toxin is also detected in clinical specimens. In some cases, isolation of BTPC in stool of individuals with symptoms of botulism provides the only evidence for laboratory confirmation in clinical specimens. Similarly, isolation of BTPC from foods provides supportive results for the identification of toxin in a contaminated food. However, isolation of BTPC only in a food source is insufficient for laboratory confirmation due to the ubiquity of such organisms in the environment. In some investigations, left over food actually consumed by patients is not available but equivalent lots may be recovered from the home or manufacturer. Therefore, subtyping methods that permit comparison of BTPC isolates from different sources have an important role in supporting epidemiological links among samples examined during a laboratory investigation of botulism especially in cases where toxin cannot be detected directly in the sample submitted for testing.

In this study, type $\mathrm{A}(\mathrm{B})$ strains isolated from different sources among separate foodborne botulism outbreaks occurring in the US were selected for study because they shared indistinguishable SmaI and XhoI PFGE patterns (Table 1). An additional type A(B) strain (CDC42961) that was unrelated to any of the botulism outbreaks was selected as an outlier. We examined the ability of high resolution subtyping methods including MLVA and genome sequence analysis (e.g. fragmented genome alignment, in slico multilocus sequence typing (MLST) analysis, reference-free SNP analysis) to distinguish these type $A(B)$ strains isolated from separate botulism outbreaks yet sharing a common PFGE pattern. This work confirms that these type $A(B)$ strains are highlyrelated and evaluates the utility of methods for clustering C. botulinum type $\mathrm{A}(\mathrm{B})$ strains isolated from separate sources during an outbreak investigation.

\section{Methods}

\section{Bacterial strains used in this study}

C. botulinum strains used in this study are indicated in Table 1. Strains were grown in Trypticase Peptone Yeast Extract (TPGY; Remel, Lenexa, KS) at $35^{\circ} \mathrm{C}$ under anaerobic conditions. Two representative strains from different sources were selected from seven separate previous laboratory investigations. An additional strain (CDC42961) unrelated to any of these investigations was also examined.

For additional studies, some isolates associated with outbreak $\# 5$ are further identified as "picks" based on their original isolation from enrichment cultures of a single sample. The pick designation following the strain number refers to the enrichment culture from which a single colony isolate was derived as follows: $\mathrm{H}=$ heated $\left(80^{\circ} \mathrm{C}, 15\right.$ minutes $)$ Cooked Meat Glucose Starch (CMGS; Remel, Lenexa, KS), T = TPGY containing $0.09 \%$ trypsin, PL = untreated (plain) CMGS. Separate single colonies from each enrichment culture type are indicated by a digit following the pick designation. All strains were coded prior to the study and managed in compliance with a human subjects exemption protocol (\#4991.0) approved by the CDC Human Research Protection Office. 
Table 1 Characteristics of strains used in this study

\begin{tabular}{|c|c|c|c|c|c|c|c|}
\hline Outbreak & Reference $^{a}$ & Strain & Source & Origin & Year isolated & Smal PFGE pattern & Xhol PFGE pattern \\
\hline \multirow[t]{2}{*}{1} & \multirow{2}{*}{ This study } & CDC28012 & Stool & Utah & 1973 & DRPS16.0001 & DRPX11.0001 \\
\hline & & CDC28023 & Chili & Utah & 1973 & DRPS16.0001 & DRPX11.0001 \\
\hline \multirow[t]{2}{*}{2} & \multirow{2}{*}{ This study } & CDC33700 & Stool & Florida & 1988 & DRPS16.0001 & DRPX11.0001 \\
\hline & & CDC33702 & Seafood pasta & Florida & 1988 & DRPS16.0001 & DRPX11.0001 \\
\hline \multirow[t]{2}{*}{3} & \multirow{2}{*}{ This study } & CDC37457 & Stool $^{\mathrm{b}}$ & Alaska & 1982 & DRPS16.0001 & DRPX11.0001 \\
\hline & & CDC37461 & Stool $^{b}$ & Alaska & 1982 & DRPS16.0001 & DRPX11.0001 \\
\hline \multirow[t]{2}{*}{4} & \multirow{2}{*}{ [18] } & CDC48719 & Stool & Georgia & 1993 & DRPS16.0001 & DRPX11.0001 \\
\hline & & CDC48761 & Swab from cutting board & Georgia & 1993 & DRPS16.0001 & DRPX11.0001 \\
\hline \multirow[t]{2}{*}{5} & \multirow{2}{*}[19,20]{} & CDC52271 & Chili sauce & Indiana & 2007 & DRPS16.0001 & DRPX11.0001 \\
\hline & & CDC52298 & Stool & Indiana & 2007 & DRPS16.0001 & DRPX11.0001 \\
\hline \multirow[t]{2}{*}{6} & \multirow{2}{*}{ This study } & CDC66088 & Stool & New York & 2011 & DRPS16.0001 & DRPX11.0001 \\
\hline & & CDC66089 & Cooked barley, sausage, cheese & New York & 2011 & DRPS16.0001 & DRPX11.0001 \\
\hline \multirow[t]{2}{*}{7} & \multirow{2}{*}{ [21] } & CDC67187 & Rectal Swab & Arizona & 2012 & DRPS16.0001 & DRPX11.0001 \\
\hline & & CDC67190 & Pruno & Arizona & 2012 & DRPS16.0001 & DRPX11.0001 \\
\hline$N A^{c}$ & This study & CDC42961 & Unknown $^{d}$ & Ecuador & 1997 & DRPS16.0001 & DRPX11.0001 \\
\hline
\end{tabular}

${ }^{a}$ Where available, references shown describe a specific outbreak or investigation.

bStool samples isolated from different individuals with foodborne botulism associated with "stink eggs".

${ }^{\mathrm{C}} \mathrm{NA}=$ not applicable; strain CDC42961 is not epidemiologically associated with any other strain examined in this study.

${ }^{\mathrm{d}}$ Strain received at CDC as a culture.

\section{MLVA}

MLVA was performed using the 15-loci scheme described by Fillo et al. [9]. Fragment analysis was performed as a separate reaction for each allele using a CEQ8000 Genetic Analysis System and the GenomeLab $^{\text {тм }}$ DNA Size Standard Kit - 600 (Beckman Coulter, Brea, CA). The largest peaks were identified in the fragment analysis and repeat units (RU) are expressed as the lowest number of intact units.

\section{Genome sequencing}

Genomic DNA was extracted from overnight TPGY cultures as described previously [10] and further purified using the DNA Clean \& Concentrator-5 kit (Zymo Research, Irvine, CA). Fragment libraries were constructed using $100 \mathrm{ng}$ of genomic DNA with the Ion Xpress $^{\text {тм }}$ Fragment Library kit and size-selection was

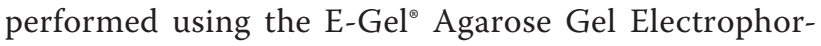
esis System (Life Technologies, Grand Island, NY). Library concentrations were determined using the Qubit $^{\oplus}$ dsDNA HS Assay (Life Technologies, Grand Island, NY) and diluted to a final concentration of 18 pM. Templates were generated using the Ion OneTouch $^{\text {Ts }} 200$ Template Kit v2 and sequencing was performed using the Ion Torrent ${ }^{\mathrm{Tm}}$ Personal Genome Machine $\left(\mathrm{PGM}^{\mathrm{Tm}}\right)$ with $314 \mathrm{v} 2$ chips. Barcoded fragment libraries of various "picks" of strains CDC 52271 and CDC 52298 were generated with the Ion Torrent-compatible Bioo Scientific (Austin, TX) NEXflex ${ }^{\mathrm{TM}}$ DNA barcodes and sequenced with a 316 v2 chip.

\section{Bioinformatic analysis}

Sequence reads were de novo assembled using MIRA v3.9.9 (performed with the Assembler plug-in on the Torrent Suite 4.0.2 software). Assembled genome metrics and GenBank accession numbers are shown in Table 2. Draft genomes were compared using Gegenees software which aligns fragments of each sequence using BLAST and generates a phylogenomic distance based on the average similarity score of fragments in pairwise alignments [11]. In silico MLST was performed using the MLST 1.7 tool available at Center for Genomic Epidemiology website (http:// cge.cbs.dtu.dk/services/MLST/) [12]. Single nucleotide polymorphism (SNP) discovery was performed on concatenated sequence data using the SNP detection program kSNP v1 [13] with a k-mer size of 21. Maximum parsimony trees were constructed in MEGA v5.2 [14] using core SNP data matrices, which include only SNPs detected at loci that were present in all genomes.

\section{Results and discussion}

Resolution of $C$. botulinum type $A(B)$ strains using MLVA

Only 2 of the 15 loci (i.e. loci 1 and 4 reported by Filio et al. [9]) examined using MLVA displayed variation in RU among the strains examined in this study (Table 3). Outbreak-related strains shared identical MLVA profiles with the exception of strains composing outbreak \#3 and outbreak \#5 which had a divergent number of repeats at various loci. In addition, the strains 
Table 2 Draft genome sequence properties

\begin{tabular}{lcccccc}
\hline Strain & \# reads assembled & Genome size $(\mathbf{M b})$ & \# contigs & N50 (kb) & Coverage $(\mathbf{X})$ & GenBank accession number \\
\hline CDC28012 & 547,889 & 3.97 & 400 & 19.1 & 29 & JFGN01000000 \\
CDC28023 & 522,829 & 3.97 & 341 & 22.3 & 27 & JFGM01000000 \\
CDC33700 & 556,551 & 3.68 & 869 & 6.4 & 30 & JFGL01000000 \\
CDC33702 & 638,235 & 3.70 & 880 & 6.2 & 37 & JFGK01000000 \\
CDC37457 & 594,516 & 3.90 & 456 & 14.5 & 34 & JFGJ01000000 \\
CDC37461 & 502,932 & 3.90 & 389 & 18.4 & 29 & JFG101000000 \\
CDC48719 & 625,316 & 3.98 & 310 & 26.1 & 29 & JFGH01000000 \\
CDC48761 & 646,981 & 3.96 & 418 & 17.0 & 36 & JFGG01000000 \\
CDC52271 & 519,456 & 3.89 & 399 & 16.9 & 28 & JFGF010000000 \\
CDC52298 & 502,934 & 3.87 & 524 & 12.0 & 28 & JFGE010000000 \\
CDC66088 & 801,959 & 3.83 & 1,086 & 5.6 & 42 & JFGD01000000 \\
CDC66089 & 458,975 & 3.44 & 1,093 & 4.1 & 26 & JFGC01000000 \\
CDC67187 & 462,117 & 3.56 & 1,079 & 4.4 & 27 & JFGB01000000 \\
CDC67190 & 594,808 & 3.73 & 1,046 & 5.0 & 35 & JFGA01000000 \\
CDC42961 & 681,808 & 3.89 & 684 & 9.4 & 35 & JFFZ010000000 \\
\hline
\end{tabular}

composing some outbreaks could not be resolved from those isolated from separate outbreaks. More specifically, the strains associated with outbreaks \#6 and \#7 shared identical MLVA profiles. Moreover, strains composing outbreak \#2 and the outlying strain CDC42961 shared the same MLVA profile. Taken together, these results demonstrate that MLVA only partially differentiates closely related type $A(B)$ strains

Table 3 MLVA results

\begin{tabular}{|c|c|c|c|c|c|c|c|c|c|c|c|c|c|c|c|}
\hline \multirow[b]{2}{*}{ Locus } & \multicolumn{15}{|c|}{ Number of repeat units (RU) } \\
\hline & 1 & 2 & 3 & 4 & 5 & 6 & 7 & 8 & 9 & 10 & 11 & 12 & 13 & 14 & 15 \\
\hline CDC28012 & 9 & $-{ }^{a}$ & 5 & 8 & 12 & 2 & 4 & 6 & 2 & 13 & 3 & 1 & 23 & 6 & 3 \\
\hline CDC28023 & 9 & - & 5 & 8 & 12 & 2 & 4 & 6 & 2 & 13 & 3 & 1 & 23 & 6 & 3 \\
\hline CDC33700 & 8 & - & 5 & 7 & 12 & 2 & 4 & 6 & 2 & 13 & 3 & 1 & 23 & 6 & 3 \\
\hline CDC33702 & 8 & - & 5 & 7 & 12 & 2 & 4 & 6 & 2 & 13 & 3 & 1 & 23 & 6 & 3 \\
\hline CDC37457 & 7 & - & 5 & 8 & 12 & 2 & 4 & 6 & 2 & 13 & 3 & 1 & 23 & 6 & 3 \\
\hline CDC37461 & 8 & - & 5 & 7 & 12 & 2 & 4 & 6 & 2 & 13 & 3 & 1 & 23 & 6 & 3 \\
\hline CDC48719 & 8 & - & 5 & 9 & 12 & 2 & 4 & 6 & 2 & 13 & 3 & 1 & 23 & 6 & 3 \\
\hline CDC48761 & 8 & - & 5 & 9 & 12 & 2 & 4 & 6 & 2 & 13 & 3 & 1 & 23 & 6 & 3 \\
\hline CDC52271 & 7 & - & 5 & 7 & 12 & 2 & 4 & 6 & 2 & 13 & 3 & 1 & 23 & 6 & 3 \\
\hline CDC52298 & 11 & - & 5 & 7 & 12 & 2 & 4 & 6 & 2 & 13 & 3 & 1 & 23 & 6 & 3 \\
\hline CDC66088 & 8 & - & 5 & 8 & 12 & 2 & 4 & 6 & 2 & 13 & 3 & 1 & 23 & 6 & 3 \\
\hline CDC66089 & 8 & - & 5 & 8 & 12 & 2 & 4 & 6 & 2 & 13 & 3 & 1 & 23 & 6 & 3 \\
\hline CDC67187 & 8 & - & 5 & 8 & 12 & 2 & 4 & 6 & 2 & 13 & 3 & 1 & 23 & 6 & 3 \\
\hline CDC67190 & 8 & - & 5 & 8 & 12 & 2 & 4 & 6 & 2 & 13 & 3 & 1 & 23 & 6 & 3 \\
\hline CDC42961 & 8 & - & 5 & 7 & 12 & 2 & 4 & 6 & 2 & 13 & 3 & 1 & 23 & 6 & 3 \\
\hline
\end{tabular}

a Deletions are indicated by a “-"symbol and not all strains associated with separate outbreaks could be resolved.

\section{Genome sequencing reveals that $C$. botulinum type $A(B)$ strains are highly-related}

Draft genome sequences from the 15 type A(B) strains were compared along with several reference genomes selected from the National Center for Biotechnology Information (NCBI) database using a fragmented alignment (Figure 1). This comparison showed that all of the type $A(B)$ genomes (including a separately sequenced type A(B) strain NCTC2916) were closely related and formed a unique clade. Within this clade, only one pair of outbreak-associated strains (CDC33700 and CDC33702) branched from a shared node indicating that such analysis is unsuitable for clustering outbreak-related strains that share a high degree of genomic similarity.

As shown in Table 4, in silico MLST analysis demonstrated that all $15 \mathrm{draft}$ genome sequences matched identically (or nearly identically) with the alleles forming ST-4 in the MLST scheme reported by Jacobson et al. [15]. In some cases, nucleotide mismatches with the best-matched allele occurred (usually in homopolymer regions) or the draft sequence did not cover the entire best-matched allele (for instance when the allele sequence was truncated by the end of a contiguous sequence). Several type A(B) strains are associated with ST-4 including the reference genome sequence of strain NCTC2916 (Table 4) and 13 out of 24 type A(B) strains examined by Jacobson et al. [15]. In that study, ST-4 was composed exclusively of type A(B) strains. 


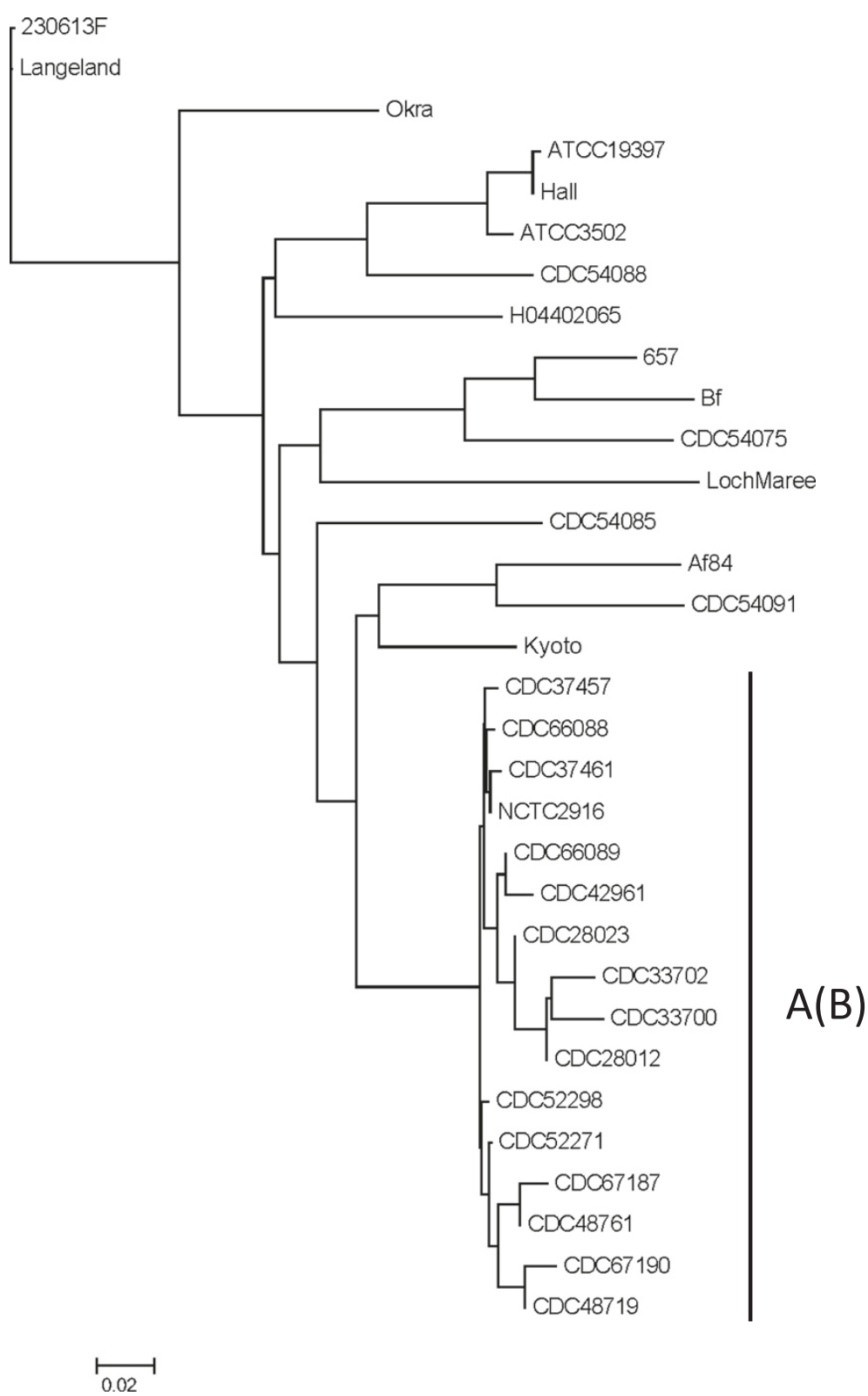

Figure 1 Phylogenomic analysis of $\boldsymbol{C}$. botulinum Group I strains. C. botulinum Group I genome sequences were compared using a fragmented alignment and a neighbor joining tree of phylogenomic distances is shown. The cluster of type A(B) strains is indicated. The accession numbers of sequences obtained from the NCBI genomes database are as follows: GenBank:NC_017297 (230613 F), GenBank:NC_009699 (Langeland), GenBank: NC_010516 (Okra), GenBank:NC_012658 (657), GenBank:NZ_ABDP00000000 (Bf), GenBank:NZ_AOSX00000000 (Af84), GenBank:NC_012563 (Kyoto), GenBank:NZ_ABDO00000000 (NCTC2916), GenBank:NC_009697 (ATCC19397), GenBank:NC_009698 (Hall), GenBank:NC_009495 (ATCC3502), GenBank: NC_017299 (H04402065), GenBank:NC_010520 (LochMaree), GenBank:AZQW01000000 (CDC54075); GenBank:AZRQ01000000 (CDC54085); GenBank: AZRR01000000 (CDC54091); and GenBank:AZRS01000000 (CDC54088).

\section{Resolution of $C$. botulinum type $A(B)$ strains using reference-free SNP analysis}

Previous work has demonstrated the utility of referencefree SNP analysis for the differentiation of C. botulinum Group I strains including those with identical BoNT subtypes [16]. This work was performed using the software program, kSNP, which can be used to identify SNPs among a set of draft and/or complete microbial genomes without the need for comparison to a reference genome [13,17]. Analysis of the $15 \mathrm{draft}$ genomes generated in this study using kSNP revealed the presence of 145 core SNPs (i.e. SNPs detected at loci 
Table 4 In silico MLST best match results

\begin{tabular}{|c|c|c|c|c|c|c|c|}
\hline \multirow[b]{2}{*}{ Locus $^{a}$} & \multicolumn{7}{|c|}{$\%$ Identity $/ \%$ Coverage of allele } \\
\hline & acek & aroE & hsp & $m d h$ & oppB & $\operatorname{rec} A$ & $r p o B$ \\
\hline Allele & 6 & 9 & 8 & 10 & 9 & 7 & 8 \\
\hline CDC28012 & $100 / 100$ & $100 / 100$ & $100 / 100$ & $100 / 100$ & $100 / 100$ & $100 / 100$ & $100 / 100$ \\
\hline CDC28023 & $100 / 100$ & $100 / 100$ & $100 / 100$ & $100 / 100$ & $100 / 100$ & $100 / 100$ & $100 / 100$ \\
\hline CDC33700 & $100 / 100$ & $100 / 100$ & $100 / 100$ & $100 / 100$ & $100 / 100$ & $100 / 100$ & $100 / 100$ \\
\hline CDC33702 & $100 / 100$ & $100 / 100$ & $100 / 100$ & $100 / 100$ & $100 / 100$ & $100 / 100$ & $100 / 100$ \\
\hline CDC37457 & $100 / 100$ & $100 / 100$ & $100 / 100$ & $100 / 100$ & $100 / 100$ & $100 / 100$ & $100 / 100$ \\
\hline CDC37461 & $100 / 100$ & $100 / 100$ & $100 / 90.4$ & $100 / 100$ & $100 / 100$ & $100 / 100$ & $100 / 100$ \\
\hline CDC42961 & $100 / 100$ & $100 / 100$ & $100 / 100$ & $100 / 100$ & $100 / 100$ & $100 / 100$ & $100 / 100$ \\
\hline CDC48719 & $100 / 100$ & $99.8 / 100$ & $100 / 100$ & $100 / 100$ & $100 / 100$ & $100 / 100$ & $100 / 100$ \\
\hline CDC48761 & $100 / 100$ & $100 / 100$ & $100 / 100$ & $100 / 100$ & $100 / 100$ & $100 / 100$ & $100 / 100$ \\
\hline CDC52271 & $100 / 100$ & $100 / 100$ & $100 / 100$ & $100 / 100$ & $100 / 100$ & $100 / 100$ & $100 / 100$ \\
\hline CDC52298 & $100 / 100$ & $100 / 100$ & $100 / 100$ & $100 / 100$ & $100 / 100$ & $100 / 100$ & $100 / 100$ \\
\hline CDC66088 & $100 / 100$ & $100 / 100$ & $100 / 100$ & 100/96.0 & $100 / 100$ & $100 / 100$ & $100 / 100$ \\
\hline CDC66089 & $100 / 100$ & $100 / 100$ & $100 / 100$ & $100 / 100$ & $99.6 / 100$ & $100 / 100$ & $100 / 100$ \\
\hline CDC67187 & $100 / 100$ & $99.8 / 100$ & $100 / 93.4$ & $100 / 100$ & $100 / 100$ & $100 / 100$ & $100 / 100$ \\
\hline CDC67190 & $100 / 100$ & $100 / 100$ & $100 / 100$ & $100 / 100$ & $100 / 100$ & $100 / 100$ & $100 / 100$ \\
\hline NCTC2916 & $100 / 100$ & $100 / 100$ & $100 / 100$ & $100 / 100$ & $100 / 100$ & $100 / 100$ & $100 / 100$ \\
\hline
\end{tabular}

aLoci refer to those genes used in the MLST scheme developed by Jacobson et al. [15].

present in all genomes). Phylogenetic analysis of these SNPs demonstrated that strains associated with each outbreak generally clustered together (Figure 2) and differed by only 3-8 SNPs. However, the strains associated with outbreak \#5 (CDC52271 and CDC52298) were more divergent and differed by 56 SNPs. Notably, strain CDC42961, which is not associated with any of the seven outbreaks examined, did not cluster with any other strain suggesting that this approach may successfully differentiate unrelated A(B) strains.

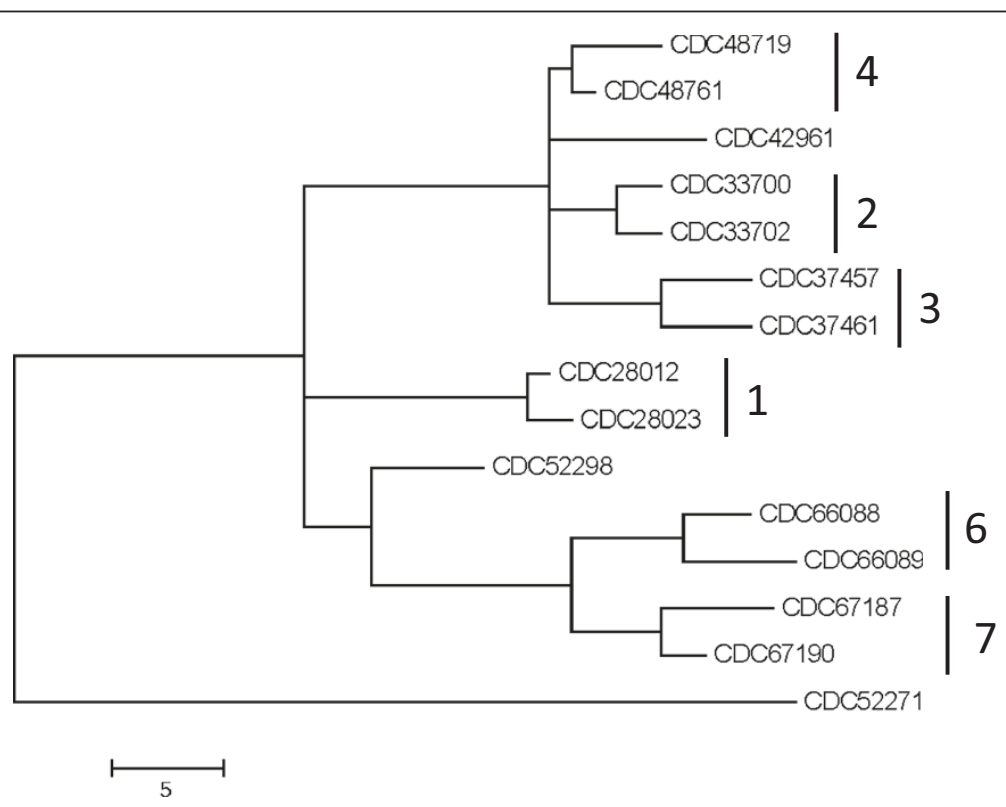

Figure 2 SNP analysis of $C$. botulinum type A(B) draft genomes sequences. Core SNPs were identified using kSNP and a maximum parsimony tree of the core SNP matrix is shown. Outbreak-related strains are indicated by outbreak number as shown in Table 1. 


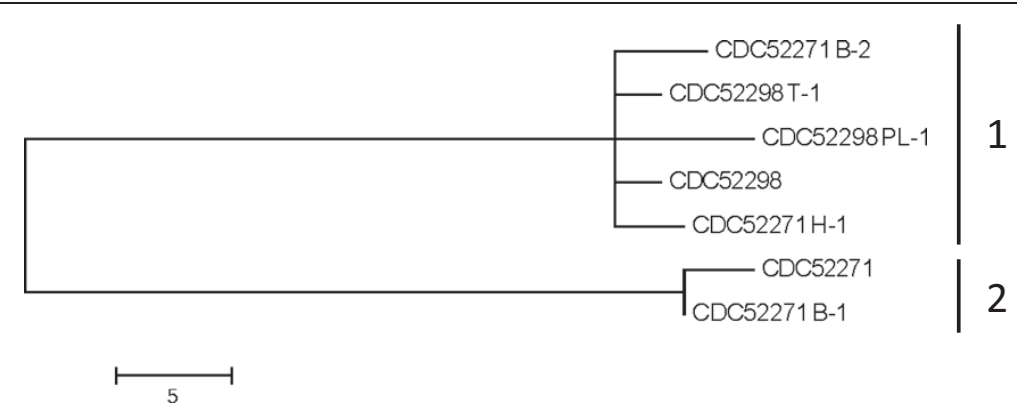

Figure 3 SNP analysis of strains isolated from outbreak \#5. Core SNPs were identified using kSNP and a maximum parsimony tree of the core SNP matrix is shown. The sequences cluster into two groups as indicated in the figure.

Resolution of multiple isolates associated with outbreak \#5 In order to better assess the genetic diversity of the strains isolated from outbreak \#5, several "picks" derived from single colonies originally isolated from different enrichment cultures of each sample (i.e. stool and food samples) were examined. Genome sequencing of these isolates revealed the presence of two groups (Figure 3). Group 1 contained the originally examined genome sequence of strain CDC52298 and two picks (CDC52298 PL-1 and CDC52298 T-1) from the same source (stool). Also included in Group 1 were picks (CDC52271 B-2, CDC52271 H-1) associated with the implicated food (chili sauce). Group 2 only contained the original strain CDC52271 genome sequence and one pick (CDC52271 B-1) associated with the same source. The number of SNPs differentiating strains associated with Groups 1 and 2 ranged from 56-63. The sequences of strains within Group 1 differed by only 4-10 SNPs and those composing Group 2 differed by only 3 SNPs. Notably, the MLVA profiles of CDC52298 PL-1, CDC52298 T-1, CDC52271 H-1, and CDC52271 B-2 were identical and also different than the profile of 52271 B-1 (data not shown), thus supporting the clustering of these isolates by SNP analysis. These data indicate that the contaminated food product contained at least two separate populations of type $\mathrm{A}(\mathrm{B})$ strains and that the strains isolated from stool of an individual who consumed the food were associated with only one these populations. These populations differ by multiple mutational events and are unlikely to be the result of recent divergence. However, more detailed comparative genomic analysis of these strains is hindered by the lack of a complete reference genome sequence.

\section{Conclusions}

The C. botulinum type $\mathrm{A}(\mathrm{B})$ strains examined in this study which shared indistinguishable PFGE profiles also shared a high degree of genomic sequence similarity. While the type $A(B)$ strains clustered together in a comparison of $C$. botulinum Group I genome sequences using a fragmented alignment, this method did not resolve strains associated with separate outbreaks. The lack of adequate resolution using this method may have been influenced by differences in the overall level of genome coverage among the de novo assembled genomes, which share little sequence variation. Strains isolated from separate foodborne botulism outbreaks also could not be distinguished by in silico MLST and only partially by MLVA.

These results suggested that genetic differences among this group of highly-related type $A(B)$ strains may be restricted to a limited number of SNPs. A complete $C$. botulinum type $\mathrm{A}(\mathrm{B})$ genome sequence is not currently available thereby preventing the ability to perform reference alignments of sequencing reads and assessment of variation at specific SNP loci. However, reference-free SNP analysis of de novo aligned draft sequences effectively resolved strains isolated from 7 separate outbreaks and an outlying unassociated type $A(B)$ strain. It is important to note that strains isolated from the same outbreak shared a low number of SNPs. It is unclear if these SNPs are associated with actual genetic variation or are artifacts of sequencing errors. Future work identifying high quality SNPs based on comparison with one or more reference genome sequences of such strains is needed.

In addition, this study demonstrates that a contaminated food product may contain multiple strains of C. botulinum some of which could be genetically distinguishable from clinical sample isolates. This finding suggests that subtyping methods may need to be applied to several isolates from the same source to ensure adequate sampling of multiple strain populations that may be present.

\section{Competing interests}

The authors declare that they have no competing interests.

\section{Authors' contributions}

BHR and $C L$ designed the study. BHR and TBS performed experiments and $\mathrm{CL}$ and SEM contributed to data analysis. BHR and TBS drafted the manuscript. All authors reviewed and approved the final manuscript. 


\section{Acknowledgements}

This publication was supported by funds made available from the Centers for Disease Control and Prevention, Office of Public Health Preparedness and Response. TBS was supported by an Oak Ridge Institute for Science and Education fellowship. The findings and conclusions in this report are those of the authors and do not necessarily represent the official position of the Centers for Disease Control and Prevention.

Received: 17 March 2014 Accepted: 10 July 2014

Published: 16 July 2014

\section{References}

1. Maslanka SE, Solomon HM, Sharma S, Johnson EA: Clostridium botulinum and its toxins. In Compendium of Methods for The Microbiological Examination of Foods. 5th edition. Edited by Doores S, Salfinger Y, Tortorello ML, Wilcke BW. Washington DC: American Public Health Association; 2013.

2. Collins MD, East AK: Phylogeny and taxonomy of the foodborne pathogen Clostridium botulinum and its neurotoxins. J Appl Microbiol 1998, 84:5-17.

3. Hill KK, Smith TJ, Helma CH, Ticknor LO, Foley BT, Svensson RT, Brown JL, Johnson EA, Smith LA, Okinaka RT, Jackson PJ, Marks JD: Genetic diversity among botulinum neurotoxin-producing clostridial strains. J Bacteriol 2007, 89:818-832.

4. Carter AT, Paul CJ, Mason DR, Twine SM, Alston MJ, Logan SM, Austin JW, Peck MW: Independent evolution of neurotoxin and flagellar genetic loci in proteolytic Clostridium botulinum. BMC Genomics 2009, 10:115.

5. Dover N, Barash JR, Arnon SS: Novel Clostridium botulinum toxin gene arrangement with subtype $\mathrm{A} 5$ and partial subtype B3 botulinum neurotoxin genes. J Clin Microbiol 2009, 47:2349-2350.

6. Franciosa G, Ferreria JL, Hatheway CL: Detection of type A, B, and E botulism neurotoxin genes in Clostridium botulinum and other Clostridium species by PCR: evidence of unexpressed type B toxin genes in type A toxigenic organisms. J Clin Microbio/ 1994, 32:1911-1917.

7. Raphael BH, Joseph LA, McCroskey LM, Lúquez C, Maslanka SE: Detection and differentiation of Clostridium botulinum type A strains using a focused DNA microarray. Mol Cell Probes 2010, 24:146-153.

8. Macdonald TE, Helma CH, Ticknor LO, Jackson PJ, Okinaka RT, Smith LS, Smith TJ, Hill KK: Differentiation of Clostridium botulinum serotype A strains by multiple-locus variable-number tandem-repeat analysis. Appl Environ Microbiol 2008, 74:875-882.

9. Fillo S, Giordani F, Anniballi F, Gorgé O, Ramisse V, Vergnaud G, Riehm JM, Scholz HC, Splettstoesser WD, Kieboom J, Olsen JS, Fenicia L, Lista F: Clostridium botulinum group I strain genotyping by 15 -locus multilocus variable-number tandem-repeat analysis. J Clin Microbiol 2011, 49:4252-4263.

10. Raphael BH, Choudoir MJ, Lúquez C, Fernández R, Maslanka SE: Sequence diversity of genes encoding botulinum neurotoxin type F. Appl Environ Microbiol 2010, 76:4805-4812.

11. Agren J, Sundström A, Håfström T, Segerman B: Gegenees: fragmented alignment of multiple genomes for determining phylogenomic distances and genetic signatures unique for specified target groups. PLOS One 2012, 7:e39107.

12. Larsen MV, Cosentino S, Rasmussen S, Friis C, Hasman H, Marvig RL, Jelsbak L, Sicheritz-Pontén T, Ussery DW, Aarestrup FM, Lund O: Multilocus sequence typing of total-genome-sequenced bacteria. J Clin Microbiol 2012, 50:1355-1361.

13. Gardner SN, Slezak T: Scalable SNP analysis of $100+$ bacterial or viral genomes. J Forensic Res 2010, 1:107.

14. Tamura K, Peterson D, Peterson N, Stecher G, Nei M, Kumar S: MEGA5: molecular evolutionary genetics analysis using maximum likelihood, evolutionary distance and maximum parsimony methods. Mol Biol Evol 2011, 28:2731-2739.

15. Jacobson MJ, Lin G, Whittam TS, Johnson EA: Phylogenetic analysis of Clostridium botulinum type A by multi-locus sequence typing. Microbiology 2008, 154:2408-2415.

16. Gonzalez-Escalona N, Timme R, Raphael BH, Zink D, Sharma SK: Whole genome SNP analysis for discrimination of Clostridium botulinum Group I strains. Appl Environ Microbiol 2014, 80:2125-2132.

17. Gardner SN, Hall BG: When whole-genome alignments just won't work: kSNP v2 software for alignment-free SNP discovery and phylogenetics of hundreds of microbial genomes. PLoS One 2013, 8:e81760.
18. Townes JM, Cieslak PR, Hatheway CL, Solomon HM, Hollowat JT, Baker MP, Keller CF, McCroskey LM, Griffin PM: An outbreak of type A botulism associated with commercial cheese sauce. Ann Intern Med 1996, 125:558-563.

19. Ginsberg MM, Granzow L, Teclaw RF, Gaul LK, Bagdure S, Cole A, Drumgoole R, Barilay EJ, Biggerstaff MS, Lynch MF, Maslanka SE, Williams IT, Juliao PC, Barton-Behravesh CB, Olson CK: Botulism associated with commercially canned chili sauce - Texas and Indiana, July 2007. MMWR Morb Mortal Wkly Rep 2007, 56:767-769.

20. Juliao PC, Maslanka S, Dykes J, Gaul L, Bagdure S, Granzow-Kibiger L, Salehi E, Zink D, Neilgan RP, Barton-Baehravesh C, Lúquez C, Buggerstaff M, Lynch M, Olson C, Williams I, Barzilay EJ: National outbreak of type A foodborne botulism associated with a widely distributed commercially canned hot dog chili sauce. Clin Infect Dis 2013, 56:376-382.

21. Briggs G, Anderson S, Komatsu K, Weiss J, Henke E, Tsang CA, Vasiq M, Rao AK, Mody RK, Lúquez C, Dykes JK, Morris JF, Anderson TC, Adams LE, Yasmin S: Notes from the field: Botulism from drinking prison-made illicit alcohol - Arizona, 2012. MMWR Morb Mortal Wkly Rep 2013, 62:88.

doi:10.1186/1471-2180-14-192

Cite this article as: Raphael et al: Distinguishing highly-related outbreak-associated Clostridium botulinum type $A(B)$ strains. $B M C$ Microbiology 2014 14:192.

\section{Submit your next manuscript to BioMed Central and take full advantage of:}

- Convenient online submission

- Thorough peer review

- No space constraints or color figure charges

- Immediate publication on acceptance

- Inclusion in PubMed, CAS, Scopus and Google Scholar

- Research which is freely available for redistribution

Submit your manuscript at www.biomedcentral.com/submit
C Biomed Central 